\title{
Sensing using differential surface plasmon ellipsometry
}

\author{
I. R. Hooper and J. R. Sambles \\ Thin Film Photonics, School of Physics, University of Exeter, Exeter, EX4 4QL, United Kingdom
}

(Received 18 March 2004; accepted 9 June 2004)

\begin{abstract}
In this work a differential ellipsometric method utilizing surface plasmons (SPs) for monitoring refractive index changes, which could be used in chemical and biological sensors, is presented. The method is based upon determining the azimuth of elliptically polarized light reflected from a Kretschmann SP system, resulting from linearly polarized light containing both $p$ and $s$ components incident upon it. The sensitivity of this azimuth to the refractive index of a dielectric on the nonprism side of the metal film is demonstrated both experimentally and theoretically. The smallest refractive index change which is resolvable is of the order of $10^{-7}$ refractive index units, although it is believed that this could be improved upon were it not for experimental constraints due to atmospheric changes and vibrations. The method requires the Kretschmann configuration to be oriented at a fixed angle, and the SP to be excited at a fixed wavelength. With no moving parts this method would be particularly robust from an application point of view. (C) 2004 American Institute of Physics. [DOI: 10.1063/1.1778218]
\end{abstract}

\section{INTRODUCTION}

The use of optically excited surface plasmon resonances (SPR) as chemical and biological sensors was demonstrated by Nylander and Liedberg in $1982,{ }^{1,2}$ and since then there has been a considerable amount of work dedicated to the development of sensors based on SPR methods. ${ }^{3}$ In general, such sensors work by detecting changes in the refractive index (or equivalent thickness of an overlayer) in the local environment of the active surface plasmon (SP) medium due to changes in the refractive index caused by some chemical or biological processes, or by the appearance of chemical and biological matter. They have also been used to observe chemical and biological reactions in real time. ${ }^{4}$

The SP is a longitudinal surface charge density oscillation at the boundary between a metal and a dielectric coupled to associated electromagnetic (EM) fields. ${ }^{5}$ Only transverse magnetic (TM) polarized incident EM fields are able to couple to the surface charge density oscillation on a flat surface since a component of the $E$-field normal to the surface is required. The nature of the $\mathrm{SP}$ is that it has exponentially decaying fields into both bounding media, with the majority of the fields being contained within the dielectric. Therefore, the SP is extremely sensitive to the refractive index of the dielectric, particularly near the SP active surface, and it is this sensitivity which makes it attractive for use in chemical or biological sensing.

The SP cannot be optically excited on a single planar metal/dielectric interface, since the wave vector matching condition requires the incident light wave vector to be greater than that available to radiation in the dielectric. There are two main methods which enable coupling of EM radiation to the SP to occur: prism coupling and grating coupling. In this paper only prism coupling will be considered, and in particular the Kretchmann method, ${ }^{6}$ although SPR sensors based on gratings have also been investigated. ${ }^{7,8}$

Since coupling to the SP occurs when the in-plane wave vector matching condition is satisfied, it will only occur at particular values of $\lambda$ (the wavelength of the incident light) and $\theta$ (the incident angle). Since a change in the refractive index of the bounding dielectric changes the wave vector of the SP, this matching condition requires there to be a corresponding change in the position of the SP in $\lambda$ and/or $\theta$. It is this sensitivity of the position of the SPR to changing refractive index which may be used to detect changes in the chemical environment of the system. The simplest method for monitoring this change involves holding $\lambda$ and $\theta$ fixed such that the reflected intensity which is measured corresponds to the edge of the SP resonance. As the refractive index of the dielectric on the nonprism side of the metal film is changed the measured reflected intensity also changes due to the shift in SPR position, and a direct relationship between the refractive index change and the intensity change can be obtained. ${ }^{1,2}$ The typical resolution for a system based upon this method is $\approx 5 \times 10^{-5}$ refractive index units (RIU). ${ }^{3}$

There are two other SPR detection methods which are prevalent in sensor applications and these involve measuring the reflected intensity as a function of $\lambda$ (Ref. 9) or $\theta$ (Ref. $10)$ and determining the position of the reflectivity minimum. The position of the minimum then changes with the refractive index of the lower medium, and the two may be correlated in order to produce a system which accurately determines the refractive index. Typical resolutions for these methods are $1.5 \times 10^{-6}$ RIU for angle measurements and $1.8 \times 10^{-6}$ RIU for wavelength measurements. ${ }^{3}$ It is also possible to use differential measurement systems which enable this resolution to be improved by an order of magnitude. ${ }^{11,12}$

More recently the possibility of using the change in phase of the reflected light through the SPR minimum to determine the refractive index of the adjacent medium has been investigated. ${ }^{13,14}$ This phase change is far more rapid as a function of either angle or wavelength than the change in the reflected intensity, thereby enabling a higher sensitivity to changes of the refractive index to be obtained. In order to measure the phase change through the SPR, heterodyne in- 
terference methods are typically used which yield sensitivities of the order of $5 \times 10^{-7}$ RIU to be obtained. However, these phase methods have the disadvantage of added complication in terms of their experimental arrangement and use.

Finally, there have been efforts to use polarization control methods to enable the SPR minimum to be narrowed ${ }^{15}$ or inverted. ${ }^{16}$ By using light polarized such that it consists of both an $s$ (TE) component, which does not excite the SP, and a $p$ (TM) component which does, and controlling the phase between them upon reflection from the metal surface, accurate control of the form of the SP reflectivity feature can be obtained. In the case of narrowing of the SPR minimum this enables a higher resolution to be obtained. The inversion method is of interest as it may be possible to track a reflectivity maximum more easily than a reflectivity minimum, enabling more accurate measurement systems, and therefore a higher sensitivity. Both of these methods involve the use of wavelength or angle determination methods for finding the SP minimum (maximum).

\section{THEORY}

The use of ellipsometric techniques to investigate SPs has not received much attention compared to the more typical techniques of angle or wavelength interrogation. The first work which suggested the use of ellipsometry for SP analysis appears to be that of Abelès in $1976 .{ }^{17}$ A more recent study linking ellipsometry and surface plasmons in regard to sensing applications is that of Westphal and Bornmann in $2002 .{ }^{18}$ The Abelès study shows that the use of ellipsometric techniques enables further information on the SPR to be gained than the more traditional techniques, including information about the phase through the SPR. This phase information is normally obtained through heterodyne interferometric methods in which the absolute phase of the p-polarized light through the SPR is obtained. This information can also be obtained through ellipsometric techniques, ${ }^{19}$ but requires comparison with a reference beam which has not undergone the phase changes which occur through the excitation of the SP. In this present study no separate reference beam is used; rather, linearly polarized light containing both $s$ - and $p$-polarization components is incident upon the SP system, with the $s$-polarized component effectively acting as the reference since it does not experience a phase change due to the excitation of a SP, whereas the $p$-polarized component does. It is only the phase difference between the reflected $s$ - and $p$-polarized components which is obtained, rather than the absolute phase as in the methods with a separate reference beam. In fact, for a SPR sensor even this phase difference is not required to produce a method with a high sensitivity to the refractive index, as will be discussed later.

Linearly polarized light incident upon a Kretschmann SP system through an input polarizer set at an arbitrary angle $\phi$ will have a $p$-component of amplitude proportional to $\cos \phi$, and an $s$ component proportional to $\sin \phi$ (if the angle of the polarizer for pure $p$-polarized light is $0^{\circ}$ ). When these two components are incident upon the SP system only the $p$-polarized component excites the SP, with the $s$-polarized component being relatively unaffected.

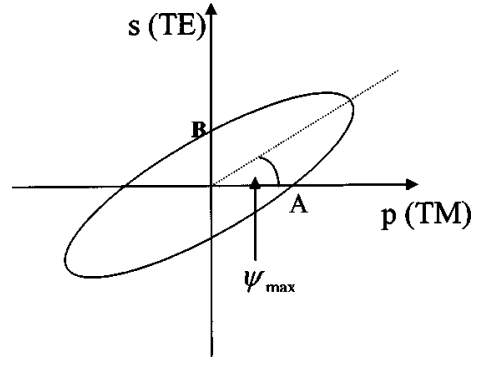

FIG. 1. A schematic of the ellipse describing the light reflected from a Kretschmann SP system with incident light consisting of both $s$ and $p$ components. $\psi_{\max }$ is the azimuth of the ellipse, with $A$ and $B$ being the purely $p$ and $s$ components.

The Kretschmann SP system may be modeled using Fresnel's equations, and in the modeling presented here a scattering matrix approach is used in order to obtain the complex amplitude coefficients for reflection from the metal film. The polarization changes caused by the incident and output faces of the prism are also taken into account in the modeling method, producing two complex amplitude coefficients: $r_{p}$ for the $p$-polarized component and $r_{s}$ for the $s$-polarized component (which are scaled in order to incorporate the different initial intensities of the $s$ and $p$ components caused by the input polarizer). These two reflection coefficients of amplitude can be written in the form

$$
\begin{aligned}
& r_{p}=A \exp \left(i \delta_{p}\right), \\
& r_{s}=B \exp \left(i \delta_{s}\right),
\end{aligned}
$$

where $A$ and $B$ are the magnitudes of the two complex amplitude coefficients, and $\delta_{p}$ and $\delta_{s}$ are their phases relative to the incident light. The phase difference between the $p$ and $s$ components is also defined as $\delta=\delta_{p}-\delta_{s}$.

If a second polarizer is now placed after the prism arrangement at some angle $\psi$ (with $0^{\circ}$ again defining the axis corresponding to purely $p$-polarized light) the total $E$ fields of the light transmitted through this polarizer are given by

$$
T=r_{p} \cos \psi+r_{s} \sin \psi,
$$

which, upon splitting into the real and imaginary components, gives

$$
\begin{aligned}
T= & A \cos \delta_{p} \cos \psi+B \cos \delta_{s} \sin \psi+i\left(A \sin \delta_{p} \cos \psi\right. \\
& \left.+B \sin \delta_{s} \sin \psi\right)
\end{aligned}
$$

with the measured intensity being given by

$$
\begin{aligned}
I= & T T^{*}=A^{2} \cos ^{2} \psi+B^{2} \sin ^{2} \psi \\
& +2 A B \cos \psi \sin \psi \cos \delta .
\end{aligned}
$$

Since, after reflection, the two orthogonal components are no longer in phase with each other the resultant $E$ fields define elliptically polarized light (Fig. 1) with the intensity for any value of $\psi$ given by Eq. (4). If the output polarizer is rotated such that the intensity is measured as a function of $\psi$, and the intensity values for $\psi=0^{\circ}$ and $90^{\circ}$ (corresponding to the values $A^{2}$ and $B^{2}$, respectively), and the angular position of the maximum of the function ( $\psi_{\max }$, the azimuth of the ellipse), are determined, it is possible to establish the phase 
between the $p$ and $s$ components resulting from the excitation of the SP. This determination of the phase difference $(\delta)$ is simply achieved by taking the differential of Eq. (4) and equating it to zero; the resultant expression can then be written as

$$
\delta=\cos ^{-1}\left(\frac{\left(B^{2}-A^{2}\right) \tan \left(2 \psi_{\max }\right)}{2 A B}\right) .
$$

Since the $s$ component does not change phase upon excitation of the SP, this corresponds to the phase change of the $p$ component through the SP, although it is not an absolute measure of the phase since the $s$ component also has a nonzero phase change relative to the incident light. There are other methods by which the absolute phase of the $p$ component through the SPR can be obtained (as described earlier), but as a measure for SPR sensors this complication is unnecessary, and in fact in this work all that is measured is the value of $\psi_{\max }\left(\right.$ or $\left.\psi_{\min }\right)$, the angle characterizing the semimajor (semiminor) of the polarization ellipse. The angle $\psi_{\max }$ is also known as the azimuth of the ellipse.

The phase of the $p$ component, and the magnitude $A$, change dramatically through the SPR. If the prism arrangement is oriented at a fixed angle such that the measured intensity is from the SPR edge on the high angle side of the intensity minimum, then as the refractive index of the lower medium is raised (meaning that the SPR moves to higher angles and passes through the angle being investigated) any change in $\psi_{\max }$ corresponds to changes in the coefficients $A$ and $\delta$. This produces a large variation in $\psi_{\max }$ or $\psi_{\min }$ for very small changes in the refractive index. It is this change in $\psi_{\max }$ or $\psi_{\min }$ as a function of the refractive index which is investigated in this paper, and which provides a promising method for SPR sensing.

\section{EXPERIMENTAL RESULTS AND DISCUSSION}

\section{A. Nondifferential: Full polarization ellipse method (large refractive index range); a demonstration of principle}

The hypotenuse face of a high index $(n=1.7305$ at $\lambda$ $=632.8 \mathrm{~nm}) 70^{\circ}$ prism is coated with a thin film $(\sim 45 \mathrm{~nm})$ of silver. A polytetrafluoroethylene container in contact with this silver film is then filled with $20 \mathrm{ml}$ of fused silica immersion fluid (from Cargille) which is chosen due to its low degradation effect on the silver film. This arrangement was placed on a computer controlled rotating table arrangement, with a HeNe laser beam $(\lambda=632.8 \mathrm{~nm}$, modulated at $1.7 \mathrm{kHz}$ using a beam chopper) incident upon the prism arrangement through a polarizer at a fixed angle. After passing through the prism arrangement the reflected light passes through a computer controlled rotating analyzer, and is detected by a photodiode connected to a lock-in amplifier (with the modulation frequency used as the reference) to give a good signal to noise ratio. The intensity could, therefore, be measured either as a function of the angle of incidence upon the prism, or as a function of the analyzer (output polarizer) angle (now defined as $\psi_{\text {out }}$ to differentiate between the analyzer and input polarizer- $\left.\psi_{i n}\right)$. This setup is shown schematically in Fig. 2.

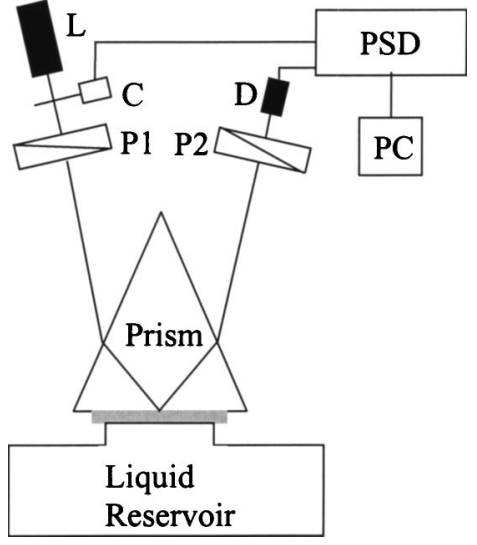

FIG. 2. A schematic of the experimental arrangement. L, HeNe laser $(632.8 \mathrm{~nm})$; C, beam chopper; P1, fixed angle polarizer; P2, rotating polarizer; D, photodiode detector; PSD, phase sensitive detector. The isosceles prism angle is $55^{\circ}$, and it has a refractive index of $n=1.7305$ (at $632.8 \mathrm{~nm}$ ).

The intensity of the laser without the SP prism arrangement is also measured in order to enable the data to be normalized, giving the absolute reflected intensity.

Initially, an angle scan is performed on the system, with the input and output polarizers both oriented at $0^{\circ}$, so that only $p$-polarized light is considered. By fitting these data to the theory produced from a multilayer optics modeling code the thickness and permittivities of the metal film are determined (Fig. 3). The parameters obtained from this fitting were found to be $\varepsilon_{\mathrm{r}}=-18.54 \pm 0.01, \varepsilon_{\mathrm{i}}=0.77 \pm 0.01$, with film thickness $d=45.0 \pm 0.1 \mathrm{~nm}$. The angular position of the SPR minimum occurred at $63.56^{\circ}$ (internal angle), and the rotating table was then oriented at a fixed angle of $63.83^{\circ}$ (internal angle), for reasons which will be made clear later.

Next the incident polarizer was oriented at $-30^{\circ}$ (this angle was chosen since it was found to give the largest change in $\psi_{\text {max } / \text { min }}$ as a function of the refractive index of the liquid under investigation, while giving a good contrast between the maximum and minimum values of the intensity as a function of $\psi_{\text {out }}$ ) and the intensity as a function of $\psi_{\text {out }}$ was obtained. It should be noted here that the form of the angular position of $\psi_{\max / \min }$ of the polarization ellipse as a function of the refractive index [given by rearranging Eq. (5)] depends upon many factors including the parameters of the silver film, the orientation of the output polarizer, the prism angles,

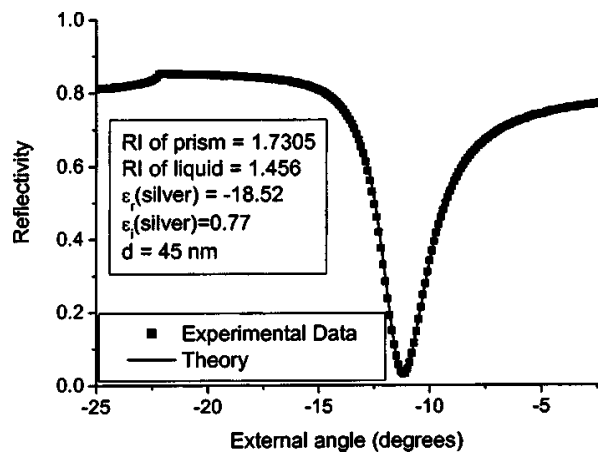

FIG. 3. The angle scan performed at the start of the liquid experiment in order to determine the parameters of the silver (inset). Both the data and theoretical fit are shown. 
(a)

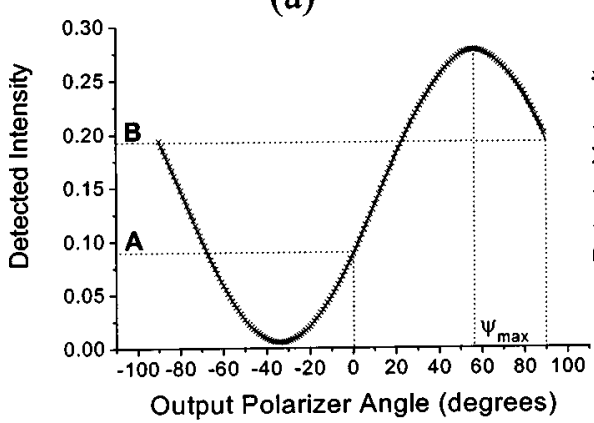

(c)

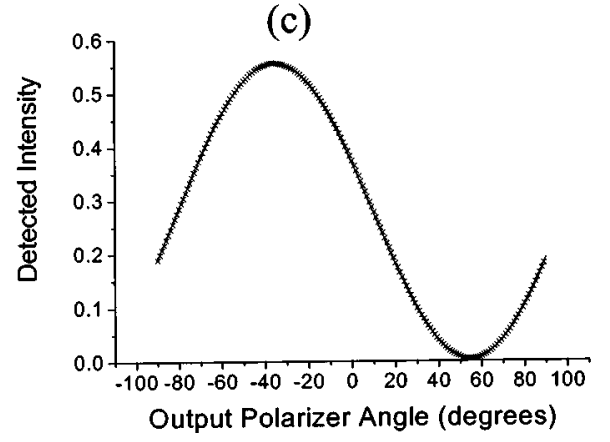

(b)

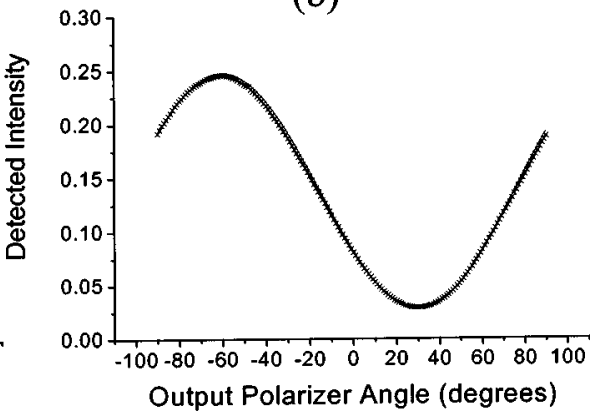

FIG. 4. The detected intensity as a function of the output polarizer angle for three different liquid mixtures corresponding to refractive indices of (a) 1.4560, (b) 1.4617, and (c) 1.4673 . Both the experimental data (crosses) and the theory (full line) are shown. The theoretical lines are obtained using the same parameters for the system as obtained from the initial angle scan. and the refractive indices of the prism and sample. Therefore, it is necessary to model the system being investigated as a whole in order to determine the ideal incident and input polarizer angles to be used (where ideal means that there is as large as possible a change in the azimuth of the polarization ellipse as a function of $n$ ). For this experiment these parameters were determined to be $\theta=63.83^{\circ}$ (internal angle) and $\psi_{\text {in }}=-30^{\circ}$.

By adding small quantities of a second, higher refractive index matching fluid ( $n=1.52$ from Cargille), the refractive index of the liquid on the nonprism surface of the metal film is increased in equal steps over a range from 1.456 (the base refractive index of the fused silica matching fluid) up to 1.4673. For each of these steps the intensity as a function of the output polarizer angle was obtained. Due to the increase in the refractive index of the liquid the SPR minimum moves through the fixed incident angle being investigated, allowing the polarization ellipse to be determined for various angles through the SPR minimum. These scans of intensity as functions of $\psi$ were all fitted to theory using the same system parameters as for the initial angle scan, with the only variable changed being the refractive index of the liquid (Fig. 4). Finally, a $p$-polarized angle scan was again performed and compared to theory in order to ensure that the parameters of the system had not changed throughout the experiment (Fig. 5).

The initial incident angle scan data in Fig. 3 shows very good agreement with theory, as do the output polarizer angle scans (Fig. 4), and the final angle scan (Fig. 5). It is also clear from Fig. 4 that there is a considerable change in the angular position giving the maximum value of the intensity $\left(\psi_{\max }\right)$ as the refractive index of the liquid is increased. In Fig. 6 a theory plot of the modulus of the differential of the intensity with respect to the output polarizer angle as a function of the output polarizer angle and the refractive index of the liquid is shown (this is plotted since the maximum of the intensity-zero in the differential-is clearer than if the intensity is plotted). Also shown on this plot are the positions of the maxima obtained from the experimental data for all values of the refractive index investigated. The change in $\psi_{\text {max }}$ over the refractive index range investigated is $\approx 80^{\circ}$, with $\psi_{\max }$ varying approximately linearly with refractive index over a range of about 0.002 RIU. It is also clear from Fig. 6 that the maximum range of the method is $\approx 1$ $\times 10^{-2} \mathrm{RIU}$, since beyond this range there is only a small change of $\psi_{\max }$ with a change of RI.

The resolution of a SP sensor system can be defined as the local slope of the response curve $\left(\Delta n / \Delta \psi_{\text {max }}\right)$ multiplied by the resolution of the measurement. For this simple measurement system $\psi_{\text {max }}$ can be measured to an accuracy of $\approx 0.2^{\circ}$. Then with the local slope of the response curve being approximately $5 \times 10^{-5} \mathrm{RIU} / \mathrm{deg}$, it yields a calculated resolution of $1 \times 10^{-5} \mathrm{RIU}$, which is worse than the other angle and wavelength methods described previously in this paper.

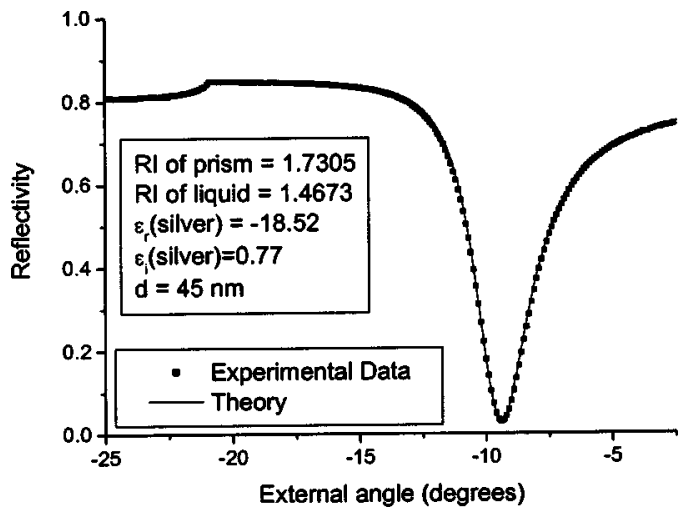

FIG. 5. The angle scan obtained at the end of the liquid experiment, with a theoretical line obtained using the same system parameters as obtained from the initial angle scan apart from a change of liquid refractive index. 


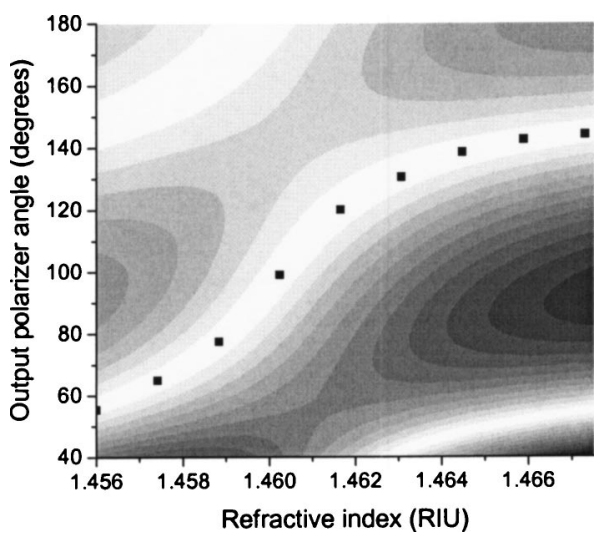

FIG. 6. A theoretical plot of the modulus of the differential of the intensity with respect to the polarizer angle (using the parameters for the system obtained from the initial angle scan), with the squares corresponding to the positions of $\psi_{\max }$ obtained from the experiment. The white in the grayscale plot corresponds to the zero in the differential, which gives the theoretical position of $\psi_{\max }$ as a function of the refractive index.

However, more accurate measuring systems for $\psi_{\max / \min }$ are described in the following sections which allow this resolution to be improved dramatically.

\section{B. Differential Methods}

\section{A differential ellipsometric method without feedback}

A Faraday rotator is a material which rotates the plane of polarization of light passing through it when it is placed within a magnetic field. The amount of polarization rotation for a given magnetic field is a property of the material, and varies linearly for small magnetic fields. By applying a sinusoidally varying field a dither in the plane of polarization is obtained. If the chopper in the experimental arrangement shown in Fig. 2 is removed, and a Faraday rotator is placed either before or after the sample with the frequency of the sinusoidally varying magnetic field used as the reference in the phase sensitive detector (PSD), the differential of the intensity as a function of $\psi_{\text {out }}$ may be obtained. The minimum position of the $\cos ^{2} \psi$ curve then appears as a zero in the differential curve $(\sin 2 \psi)$, which allows the use of a feedback loop to change the output polarizer angle in order to compensate for any rotation of the polarization from the SP arrangement due to a change in the refractive index of the sample. In addition, as well as applying a sinusoidally timevarying magnetic field to the Faraday rotator, a quasistatic field can also be applied in order to give a rotation of the plane of polarization to compensate for the change of refractive index. Plots of changes in $\psi_{\text {out }}$ (or of the quasistatic magnetic field applied to the Faraday rotator) indicate any change in the refractive index of the sample. There are two significant benefits to using this differential technique. First the differential signal varies its magnitude faster as a function of $\psi_{\text {out }}$ than does the nondifferential signal allowing greater sensitivity to any changes in the orientation of the plane of polarization, with the change in the measured signal being approximately linear around the zero point. Second, since the zero in the signal is independent of the intensity of the laser being used, fluctuations in the laser intensity have no effect on the data if a feedback loop locked to the zero is being used. It is also possible to not use a feedback loop and simply monitor the signal changes around the zero point of the differential curve. This gives the benefits of the increased sensitivity of the differential method, but still suffers from variations in the laser intensity. It is this method which is presented in this section with the feedback method discussed last.

In this experiment the Faraday rotator is a $5 \mathrm{~mm}$ diameter, $3.6 \mathrm{~cm}$ long rod of terbium gallium garnet (TGG) $\left(\right.$ Verdet constant $=-134 \mathrm{rad} \mathrm{T}^{-1} \mathrm{~m}^{-1}$ ) placed within two 500 turn coils, one outside the other. A $210 \mathrm{~Hz}$ sinusoidal ac current is passed through one of these coils creating a time varying magnetic field at the TGG rod, with the option of using a dc current through the second coil to give a compensation (quasistatic) magnetic field at the TGG rod providing feedback. The time varying magnetic field gives the required sinusoidal dither of the plane of polarization needed to obtain the differential of the $\cos ^{2} \psi$ intensity variation with $\psi_{\text {out }}$.

In order to determine the smallest polarization rotation which could be measured using the differential system with the TGG Faraday rotator the sample was removed and all components placed in line with each other. The output polarizer angle was set orthogonal to the input polarizer such that the differential signal read as close to zero as was possible. By rotating the output polarizer by small increments and measuring the signal and noise of the signal the smallest rotation measurable could be determined. If the smallest significant change in the signal is considered as being given by $2 \times$ the standard deviation of the noise (in other words $95 \%$ of the points in the data set would occur within this value unless a rotation has occurred), then the smallest rotation measurable is $\approx 2 \times 10^{-5} \mathrm{deg}$. As described earlier, the resolution of a SP sensor system can be defined as the local slope of the response curve $\left(\Delta n / \Delta \psi_{\min }\right)$ multiplied by the resolution of the measurement. Typically, local slopes of the response curves are of the order of $5 \times 10^{-5} \mathrm{RIU} / \mathrm{deg}$ (depending on the precise system being investigated). If the smallest measurable polarization rotation is $2 \times 10^{-5} \mathrm{deg}$ this would imply that refractive index changes of the order of 1 $\times 10^{-9}$ RIU could be measured. In reality this is not possible due to increased noise from the sample (vibrations) and changes in the atmospheric conditions (temperature and pressure) which are commonly thought to be a problem at below $\approx 1 \times 10^{-7}$ RIU.

In this experiment a $54 \mathrm{~nm}$ silver film was deposited onto a $45^{\circ} \mathrm{SiO}_{2}$ prism by thermal evaporation. A $p$-polarized reflectivity scan was performed on the sample and fitted to theory in order to characterize the silver film (Fig. 7). From this, modeling was performed in order to determine the ideal angle setting of the sample and input polarizer to give the steepest $\Delta n / \Delta \psi_{\min }$ curve whilst maintaining a sufficiently large difference between the maximum and minimum of the intensity as a function of $\psi_{\text {out }}$ curve to enable a strong differential signal to be maintained. For this system the modeling showed that the SP arrangement should be oriented at an internal angle of $45.045^{\circ}\left(0.124^{\circ}\right.$ external angle $)$ with the 


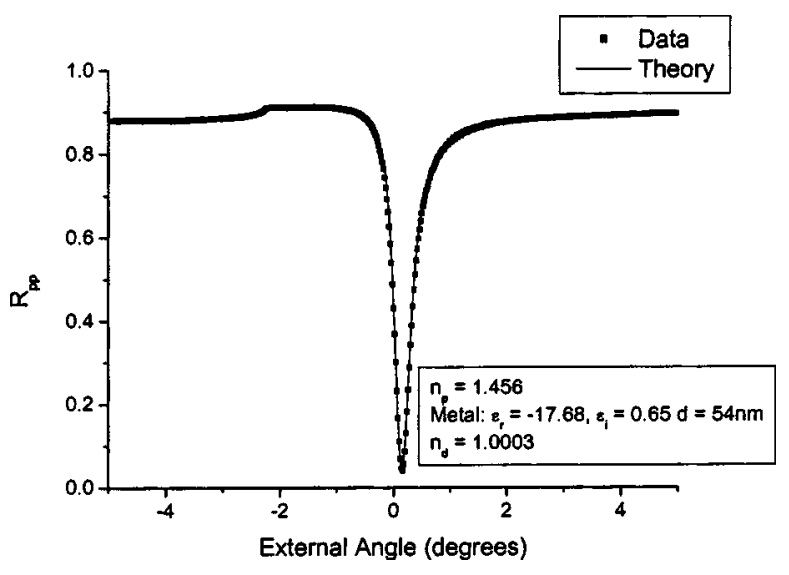

FIG. 7. The measured data and comparison with theory of the surface plasmon resonance used for the differential experiment. The silver film parameters obtained are shown, and these were subsequently used in comparing the data obtained from the differential experiments with theory.

output polarizer oriented at an angle of $30^{\circ}$ from that which allows pure $p$-polarized light to pass. The arrangement used for the experiment is shown in Fig. 8.

The differential signal from the PSD was then measured as a function of time as a gas mix adjacent to the SP-active interface was changed from pure nitrogen to pure argon with the amount of argon in the mix then being changed in $\approx 10 \%$ steps while reverting to pure nitrogen between steps. Note, there was also an approximately linear trend in the data due to long term changes in atmospheric conditions which has been removed from the data, which is shown in Fig. 9.

The signal change arising from a $1^{\circ}$ rotation of the polarization ellipse was determined by rotating the output polarizer by $1^{\circ}$, and was found to be $5.6 \mathrm{mV}$. Since the change in signal is approximately linear in the region of the zero in the differential intensity curve this value was used to convert the measured signals shown in Fig. 9 to rotations of the polarization ellipse, which could then be compared to theory. This is shown in Fig. 10. There are two theory lines shown in Fig. 10, one for an incident external angle of $0.124^{\circ}$, and one for an incident external angle of $0.122^{\circ}$. Even though the incident angle used in the experiment was intended to be $0.124^{\circ}$, it is clear that the model theory corresponding to an

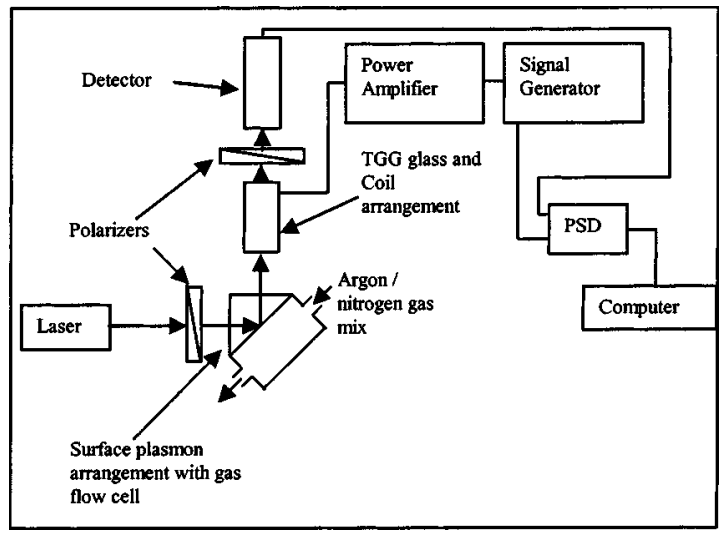

FIG. 8. A schematic of the experimental arrangement used in both differential experiments. Note, the refractive index of the prism is now 1.456 , with the prism angle being $90^{\circ}$.

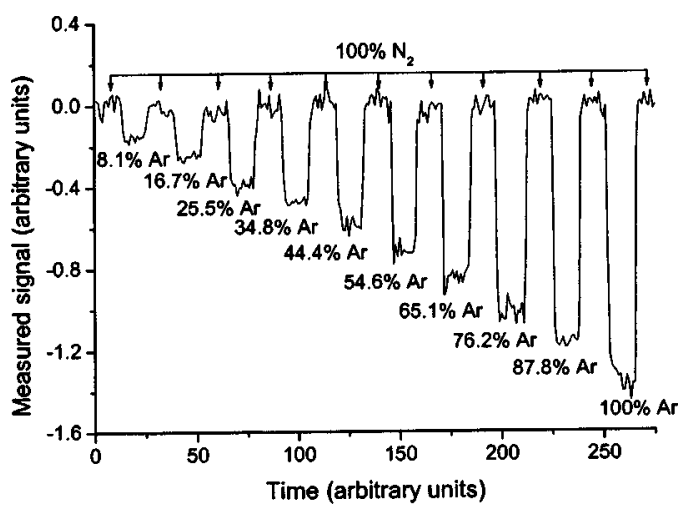

FIG. 9. The measured signal obtained for the nonfeedback differential experiment as the proportion of argon in the argon-nitrogen mix is changed.

incident angle of $0.122^{\circ}$ compares better with the data. This is a result of the difficulty in orienting the sample to this precision. Also, the majority of the discrepancy between the theory and the data is due to the inaccuracy in mixing the two gases. It is clear from Fig. 9 that a refractive index change due to the presence of $\approx 4 \%$ argon is the smallest measureable by this experiment, and this corresponds to a refractive index change of $\approx 5 \times 10^{-7}$ RIU. Due to detector limitations (the light threshold before overloading) the RI range is reduced to $\approx 1 \times 10^{-4} \mathrm{RIU}$, as opposed to the 1 $\times 10^{-2}$ RIU for the experiment in the preceding section.

The resolution obtained from the experiment is approximately three orders of magnitude worse than would be expected when compared to the theoretical best obtained by multiplying the local slope of the response curve by the smallest resolvable rotation change without the sample present. There are three main sources of noise which limit the resolution: atmospheric changes, vibrations, and fluctuations in the gas mixture. The fluctuations in the gas mixture are quite significant, although it is not certain whether they are due to temperature or flow (pressure) fluctuations. This is clear when the noise in the data when gases are flowing through the cell is compared to that when the flow cell is sealed. The noise when the cell is sealed is approximately one half to one quarter of that found when the gases are

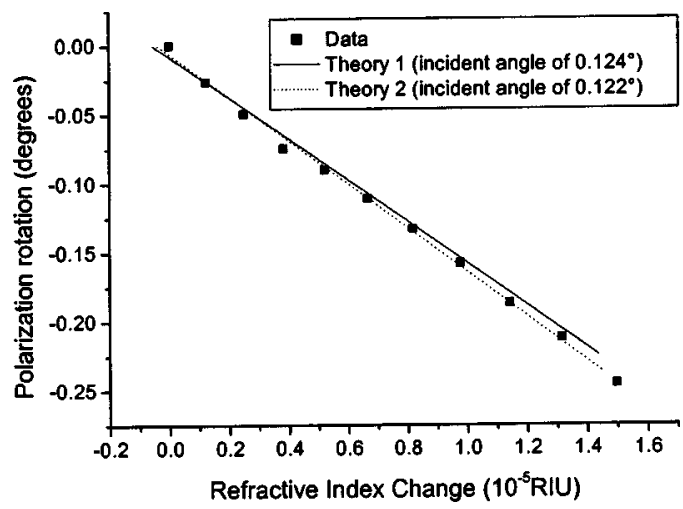

FIG. 10. A comparison of the data obtained from Fig. 9 (after conversion to polarization rotation) with the theory obtained using the parameters obtained from Fig. 7. Two theory lines are shown: one for an incident external angle of $0.124^{\circ}$ (the intended incident angle), and one for the best comparison with the data at an incident external angle of $0.122^{\circ}$. 
flowing, and clearly this could be eradicated with a more sophisticated gas mixing/supply process. The atmospheric changes tend to be long period variations due to changes in temperature and pressure which for a system such as the one investigated could only be eradicated through operation in an atmosphere-controlled room. A more significant contribution to the short term are any vibrations of the sample. Since the SPR is narrow in angle, any vibration causing the light to impinge upon the sample at a slightly altered angle has a large effect on the measured signal. In this experiment the sample is mounted on a rotating table, in order to allow the incident angle to be set accurately, and, even though the entire system is mounted upon a vibration-damped optical table and the sample is fixed rigidly, some vibration will still occur. One possible means of reducing the effect of these vibrations for chemical and biological sensors, where specific molecular interactions through specific binding events to molecules coating the metal film give rise to the SPR change, is to use pixelated systems where one region (pixel) of the silver film is not coated with the binding molecules and to use this uncoated pixel as a reference. This would then remove a large proportion of the atmospheric and vibrational noise giving an increased sensitivity to local refractive index changes.

\section{A differential ellipsometric method with feedback}

In this experiment the same experimental arrangement as that described in the preceding section (shown in Fig. 8) is used, but in this case a feedback circuit to the compensation second coil surrounding the Faraday rotator is used in order to lock to the zero in the differential signal. This is achieved through "labview," which outputs a voltage to a voltagecontrolled current source, the output of which was connected to this second coil. The voltage dependence of the rotation of the plane of polarization by the Faraday rotator was determined, enabling the voltage required to keep the differential signal at zero to be converted to a rotation of the polarization ellipse, and hence the position of $\psi_{\min }$ could be accurately determined.

An experiment involving changing the composition of a mixture of argon and nitrogen gases, as in the preceding section, was performed and the results of this are shown in Fig. 11, with the corresponding comparison of the polarization rotation with theory shown in Fig. 12. By comparing the results in Fig. 11 with those for the nonfeedback differential experiment in Fig. 9 it is clear that some of the noise has been removed, which enables even smaller percentages of argon in nitrogen to be resolved. This reduction in noise is most likely due to the fact that any change in the intensity of the illuminating light source will not have any influence in the measured signal since this remains locked at zero. However, it is also possible that some of the reduction in noise is due to the slightly different data averaging methods needed for the two experiments. From Fig. 11 it appears that argon percentages below $2 \%$ may be resolved corresponding to a refractive index resolution of better than $2 \times 10^{-7}$ RIU. This is comparable to other differential experiments involving angle or wavelength interrogation of SPRs, and also to the heterodyne phase methods. The comparison to theory shown

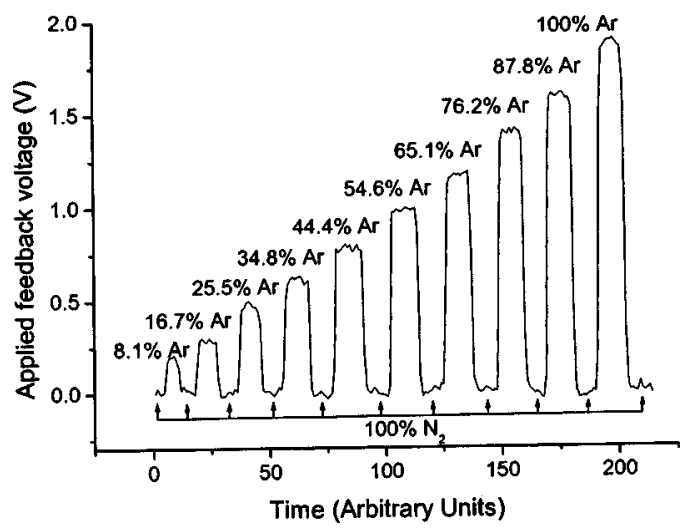

FIG. 11. The measured signal obtained for the feedback differential experiment as the proportion of argon in the gas mix was changed. The sign of the voltage is opposite to that in Fig. 9 because the phase of the reference signal with respect to the measured signal was $180^{\circ}$ different in this experiment. The choice of a positive direction is arbitrary.

in Fig. 12 shows good agreement, although, as with the nonfeedback differential experiment, the theory obtained for an incident angle of $0.122^{\circ}$ shows better agreement than that for the intended incident angle of $0.124^{\circ}$, with the majority of the discrepancy between theory and data being due to the inaccuracy in the gas mixing process.

\section{CONCLUSIONS}

In this paper an ellipsometric method for using SPRs in chemical and biological sensing has been presented. The method depends upon determining the azimuth of elliptically polarized light resulting from the reflection of light consisting of both $s$ and $p$ components from a Kretchmann SP system. The azimuth angle of this ellipse is related to the phase difference between the $s$ and $p$ components of the reflected light, and since the phase of $p$-polarized light undergoes a sharp and pronounced change through the SPR it is found that the azimuth angle of the polarization ellipse also shows a pronounced variation through the SP resonance. Due to the sensitivity in the angular position of the SPR with the refractive index of a liquid bounding the SP active medium, this azimuth-determination method provides a sensitive measure

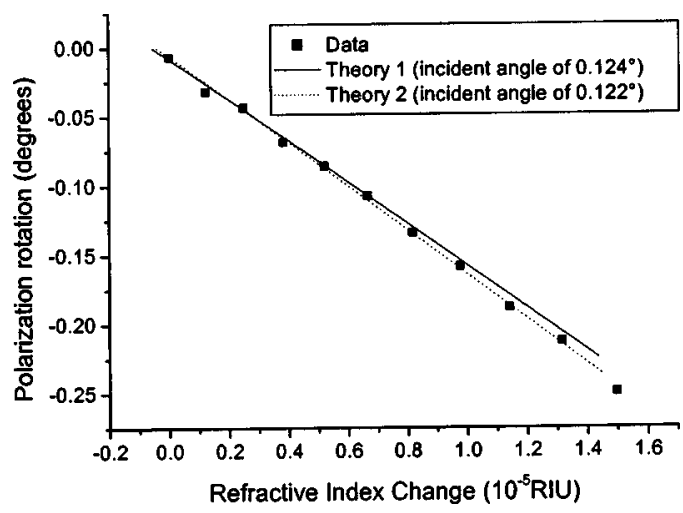

FIG. 12. A comparison of the data obtained from Fig. 11 (after conversion to polarization rotation) with the theory obtained using the parameters obtained from Fig. 7. Two theory lines are shown: one for an incident external angle of $0.124^{\circ}$ (the intended incident angle), and one for the best comparison with the data at an incident external angle of $0.122^{\circ}$. 
of the refractive index of the sample. Three experiments to monitor this change in the azimuth position of the polarization ellipse have been described in detail, one nondifferential and two differential. The nondifferential method is shown as a demonstration of the principle and has a refractive index resolution of only $\approx 1 \times 10^{-5} \mathrm{RIU}$, which is an order of magnitude worse than given by typical methods using angle or wavelength interrogation of the SPR. However, this resolution is simply limited by the determination of the angular rotation of the polarization ellipse. In the differential methods, a Faraday rotator is used to give a dithered plane of polarization; this leads to an increased refractive index sensitivity. With a Faraday rotator as the polarization modulator, and without a feedback mechanism, the refractive index resolution is $5 \times 10^{-7} \mathrm{RIU}$, whereas with feedback the resolution is $2 \times 10^{-7}$ RIU. These figures correspond to an angular resolution of the polarization azimuth of between $0.004^{\circ}$ and $0.01^{\circ}$. This is limited by noise in the signal which, for the Faraday rotator, is believed to be caused almost entirely by atmospheric variations and vibrations. Reductions in noise would obviously allow much smaller refractive index changes to be measured. The maximum range of refractive index which can be measured using this technique is $\approx 1$ $\times 10^{-2} \mathrm{RIU}$, although for higher sensitivity this range is reduced due to detector limitations.

The fact that these differential methods have no moving parts, are relatively simple, and are at least as sensitive as the commonly used angle and wavelength determination methods means that they may be attractive as robust biological and chemical sensing elements.

\section{ACKNOWLEDGMENTS}

The authors would like to acknowledge the support of the Engineering and Physical Sciences Research Council. This work was performed as part of a collaborative project with M. Somekh and M. Pitter of Nottingham University, UK.

${ }^{1}$ C. Nylander, B. Liedberg, and T. Lind, Sens. Actuators 3, 79 (1982).

${ }^{2}$ B. Liedberg, C. Nylander, and I. Lundström, Sens. Actuators 4, 299 (1983).

${ }^{3}$ J. Homola, S. S. Yee, and G. Gauglitz, Sens. Actuators B 54, 3 (1999).

${ }^{4}$ I. Lundström, Biosens. Bioelectron. 9, 725 (1994).

${ }^{5} \mathrm{H}$. Raether, Surface Plasmons on Smooth and Rough Surfaces and on Gratings (Springer, Berlin, 1988).

${ }^{6}$ E. Kretschmann and H. Raether, Z. Naturforsch. A 23A, 2135 (1968).

${ }^{7}$ D. C. Cullen and C. R. Lowe, Biosensors 3, 211 (1987).

${ }^{8}$ P. S. Vukusic, G. P. Bryan-Brown, and J. R. Sambles, Sens. Actuators B 8, 155 (1992).

${ }^{9}$ L. M. Zhang and D. Uttamchandani, Electron. Lett. 23, 1469 (1988).

${ }^{10}$ K. Matsubara, S. Kawata, and S. Minami, Appl. Opt. 27, 1160 (1988).

${ }^{11}$ M. J. Jory, G. W. Bradberry, P. S. Cann, and J. R. Sambles, Meas. Sci. Technol. 6, 1193 (1995)

${ }^{12}$ C. E. H. Berger and J. Greve, Sens. Actuators B 63, 103 (2000).

${ }^{13}$ S. G. Nelson, K. S. Johnston, and S. S. Yee, Sens. Actuators B 35, 187 (1996).

${ }^{14}$ P. I. Nikitin, A. A. Beloglazov, A. V. Kabashin, M. V. Valeiko, and V. E. Kochergin, Sens. Actuators B 54, 43 (1999).

${ }^{15}$ A. V. Kabashin, V. E. Kochergin, and P. I. Nikitin, Sens. Actuators B 54, 51 (1999).

${ }^{16}$ J. Homola and S. S. Yee, Sens. Actuators B 51, 331 (1998).

${ }^{17}$ F. Abelès, Surf. Sci. 56, 237 (1976).

${ }^{18} \mathrm{P}$. Westphal and A. Bornmann, Sens. Actuators B 84, 278 (2002).

${ }^{19}$ V. Vaicikauskas, J. Bremer, O. Hunderi, R. Antanavicius, and R. Januskevicius, Thin Solid Films 411, 262 (2002). 